\section{How to Calculate the Temperature Rise Due to Beam Heating}

William F. Tivol, Wadsworth Center

The temperature of a specimen rises when the electron beam interacts with it, producing ionization and excitation of atoms and breaking molecular bonds. Energy loss in bulk materials is ultimately converted to heat, but for small particles some of the energy escapes. Of the energy lost by the electrons in the incident beam, that which is absorbed by the specimen and degraded to heat includes oscillations of valence electrons, the kinetic energy of low-energy secondary electrons, and radiationless recombination of ionized atoms or molecules. Energy not absorbed includes brehmsstrahlung, characteristic $x$-rays, and the kinetic energy of higher-energy secondary electrons. Glaeser (1979) estimated that $50 \%$ of the energy loss is confined to a distance of about $5 \mathrm{~nm}$ from the track of the incident electron, while the other $50 \%$ is largely due to secondary electrons having 0.5 to $5 \mathrm{keV}$ kinetic energy. Since the range of a $10 \mathrm{keV}$ electron in nickel is about $0.5 \mu \mathrm{m}$ (Berger and Seltzer, 1982), much of this energy is converted to heat as well. In the following, then, I will assume that any energy loss not resulting in the production of electromagnetic radiation ( $\mathrm{x}$-rays) will be absorbed and will contribute to heating.

In order to calculate the heat input, one needs to have the stopping power of the material for electrons of the appropriate energy. For $200 \mathrm{keV}$ electrons in nickel that would be about $1.9 \mathrm{MeV} \mathrm{cm} / \mathrm{g}$ (Berger and Seltzer, 1982). This figure is the collision stopping power. Using the simplifying assumption that the energy absorbed is the same for all points within the particle, the total heat input per unit time, $\mathrm{H}$, is calculated by multiplying the stopping power by the density to get energy deposited per unit length for each electron, multiplying by the electron flux, then integrating over the volume, $V$, of the particle (equivalent to integrating along the path for each possible path, assuming that the path length is the same as that for an undeflected electron). This gives the energy deposited per electron per unit length as $\left(8.9 \mathrm{~g} / \mathrm{cm}^{3}\right) \cdot(1.9 \mathrm{MeV} \mathrm{cm} / \mathrm{g})=(17 \mathrm{MeV} / \mathrm{cm})$ $=(1.7 \mathrm{eV} / \mathrm{nm})$, or $H=(1.7 \mathrm{eV} / \mathrm{nm}) \cdot \mathrm{V} \cdot \mathrm{J}$, where $\mathrm{J}$ is the electron flux. For a beam current of $10^{3}$ electrons/s $\cdot \mathrm{nm}^{2}$, and a particle with a $1 \mu \mathrm{m}$ radius, the input heat is $(4 \pi / 3) \cdot 10^{9} \mathrm{~nm}^{3}$. $1.7 \mathrm{eV} / \mathrm{nm} \cdot 10^{3}$ electrons $/ \mathrm{s} \cdot \mathrm{nm}^{2}=7 \cdot 10^{12} \mathrm{eV} / \mathrm{s}$.

The loss is through radiation and conduction. The former

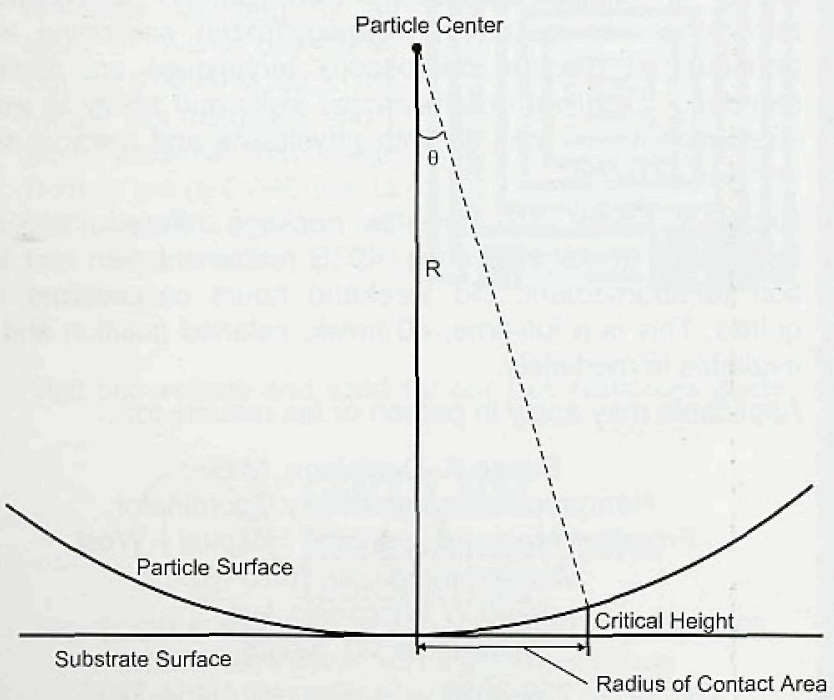

depends on the total surface area, and the latter is a function of contact area (among other variables). The particles can probably be treated as black bodies. At most a constant, $\mathrm{k}_{\mathrm{g}}$, would be put into the black-body equation; the particle is then a gray body. The heat radiated is $\mathrm{kg}_{\mathrm{g}} \cdot \sigma \cdot \mathrm{A} \cdot \mathrm{T}_{\mathrm{p}}{ }^{4}$ for a particle of surface area $A$, where $\sigma$ is $5.67 \cdot 10^{-5} \mathrm{erg} / \mathrm{s}$ for $\mathrm{A}$ in $\mathrm{cm}^{2}$ and $T_{p}$ in Kelvins (Handbook of Chemistry and Physics ). Of course, the particle also absorbs radiation at the rate $k_{g} \cdot \sigma \cdot A \cdot T_{A}{ }^{4}$, where $T_{A}$ is the ambient temperature, so the net radiation loss is $k_{g} \cdot \sigma \cdot A \cdot\left(T_{p}^{4}\right.$ $T_{A}{ }^{4}$ ). For $k_{g}=1$ and a particle with a radius of $1 \mu \mathrm{m}$ at $T_{A}=300$ $K$, the radiative heat loss at the melting temperature of nickel $(1726 \mathrm{~K})$ is $63 \mathrm{erg} / \mathrm{s}$ or $4.0 \cdot 10^{13} \mathrm{eV} / \mathrm{s}$.

The conductive loss is more difficult to calculate. Assuming that any part of the particle closer to the substrate surface than some critical height, $h_{c}$ will be in contact with the substrate -- i.e., thermal vibrations of atoms of the particle can be efficiently transmitted to atoms of the substrate -- the contact area for a particle of radius $\mathrm{R}$ can be calculated. The geometry is shown in the figure. Letting the radius of the contact area be $R_{c}$, we have, with very little work, $R_{c}=R \cdot \sin \theta$ and $h_{c}=R(1-\cos \theta)$. Eliminating $\theta$ gives $R_{c}=\left(2 R h_{c}-h_{c}^{2}\right)^{1 / 2}$. The contact area, $A_{c}$, is $A_{c}=\pi\left(2 R h_{c}-h_{c}^{2}\right)$.

If the contact is with a thick enough substrate (as in the SEM), the conductive loss will be proportional to the contact area, but if the substrate is too thin (as in the TEM), the heat conducted into it will have to diffuse away from the particle, and the loss will be proportional to the circumference of the contact area times the film thickness. Guessing that $h_{c}$ is about the sum of the Van der Waals radii of the atoms of the particle and substrate (read off of any chart which has bars representing atomic sizes on it, such as the NSRDS (National Standard Reference Data System) chart, 1979 revised edition), and hoping that the effects of surface roughness will not be significant, this gives for a $1 \mu \mathrm{m}$ nickel particle on aluminum, $A_{c}=4 \cdot 10^{3} \mathrm{~nm}^{2}$, and for the same particle on a carbon film, the circumference of the contact area is $2 \cdot 10^{2} \mathrm{~nm}$, and for a $1 \mathrm{~nm}$ film thickness, the area through which heat is conducted is $2 \cdot 10^{2} \mathrm{~nm}^{2}$.

The conductive loss is $H_{c}=K \cdot A_{c} \cdot d T / d z$, where $K$ is the thermal conductivity and $A_{c}$ is the appropriate area. If the center of the particle is at a temperature $T_{\rho}$ and the substrate is at ambient temperature $\left(T_{S}=T_{A}\right)$, the thermal gradient can be approximated as linear, giving $H_{c}=K \cdot A_{c} \cdot\left(T_{p}-T_{s}\right) / R$. The thermal conductivity of carbon varies greatly with its form. Using the value for graphite, and the better defined values for the conductivities of nickel and aluminum (Handbook of Chemistry and Physics), and taking $T_{s}=300 \mathrm{~K}$ and $T_{p}=1726 \mathrm{~K}$, for the usual $1 \mu \mathrm{m}$ nickel particle the conductive heat loss is about $0.6 \mathrm{~mW}$ for the aluminum substrate and about $30 \mu \mathrm{W}$ for the carbon substrate. This converts to about $4 \cdot 10^{15} \mathrm{eV} / \mathrm{s}$ for aluminum and $2 \cdot 10^{13} \mathrm{eV} / \mathrm{s}$ for carbon. Although several questionable assumptions were made in this derivation, the conductive loss is predicted to be larger than the radiative loss for contact with a bulk substrate, and about equal for contact with a thin film under the assumed conditions. Both losses are predicted to be larger than the heat input, which implies that for the example beam current, the temperature of a 1 $\mu \mathrm{m}$ particle would not approach the melting temperature of nickel.

As the radius of the particle increases, the heat input rises faster ( $\propto \mathrm{R} 3$ ) than either loss process, and radiative loss rises faster ( $\propto R 2$ ) than conductive loss, which rises very slowly ( $\propto R$ or $\mathrm{R}^{1 / 2}$ ). This predicts that a nickel particle with a radius of several micrometers on a thin carbon substrate will melt in an electron beam whose flux is $10^{3}$ electrons $/ \mathrm{s} \cdot \mathrm{nm}^{2}$. 


\section{Harress trie powyer of WDX}

\section{yit'́n J」さA Wave}

We all know that Wavelength Dispersive $\mathrm{X}$-ray (WDX)

microanalysis is a powerful technique, but it can seem like

a real struggle.

With INCAWave, you now have the power of WDX with all the ease and speed of the INCA platform.

For more information on INCA visit our website today at www.oxford-instruments.com/mag Or call us on:

USA 1 (978) 3699933 ,

UK +44 (0) 1494442255 ,

France (33)-01-69 852521 ,

Germany (49) 06122 937-176,

INCA is a trademark of Oxford Instruments

Scandinavia (46) 859072550

Australia (61) 294846108 ,

Japan (81) 3-5245-3591.

Singapore (65) 337-6848,

China (86) 1068330336.

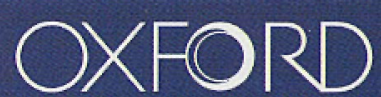

I N S T R U M EN T S 


\section{How to Calculate the Temperature Rise Due to Beam Heating \\ Continued from page 24}

As the beam current increases, the heat input increases proportionally. The conductive loss increases in proportion to the temperature rise, which in turn is proportional to the heat input, so the steady-state temperature should be linear with the beam current for the case where radiative heat loss is negligible. Radiative loss, however, is a steeply increasing function of temperature, so when radiation is the dominant loss process, the temperature increases only very slowly with beam current. This predicts that an electron flux of $10^{4}$ electrons $/ \mathrm{s} \cdot \mathrm{nm}^{2}$ would melt a $1 \mu \mathrm{m}$ nickel particle on a thin carbon substrate. Melting a nickel particle on an aluminum substrate would require a much larger particle and/or beam current (unless the thermal contact between particle and substrate was so poor that conduction losses were much less than l've calculated).

Finally to calculate the steady-state temperature of a particle under the electron beam, use the fact that at steady state the heat input is equal to the heat lost.

$\left.P \quad \frac{d E}{d x}\right|_{\text {col }} \frac{4 \pi}{3} \quad R^{3} J=k_{g} \sigma 4 \pi R^{2}\left(T^{4}{ }_{p}-T_{s}^{4}\right)+K A_{c} \frac{T_{p}-T_{s}}{R}$

Where: $p$ is the density

$\left.\frac{d E}{d x}\right|_{\text {col }}$ is the collision stopping power

$\mathrm{k}_{\mathrm{g}}$ is a "gray body" constant

$\sigma$ is the Stefan-Boltzmann constant

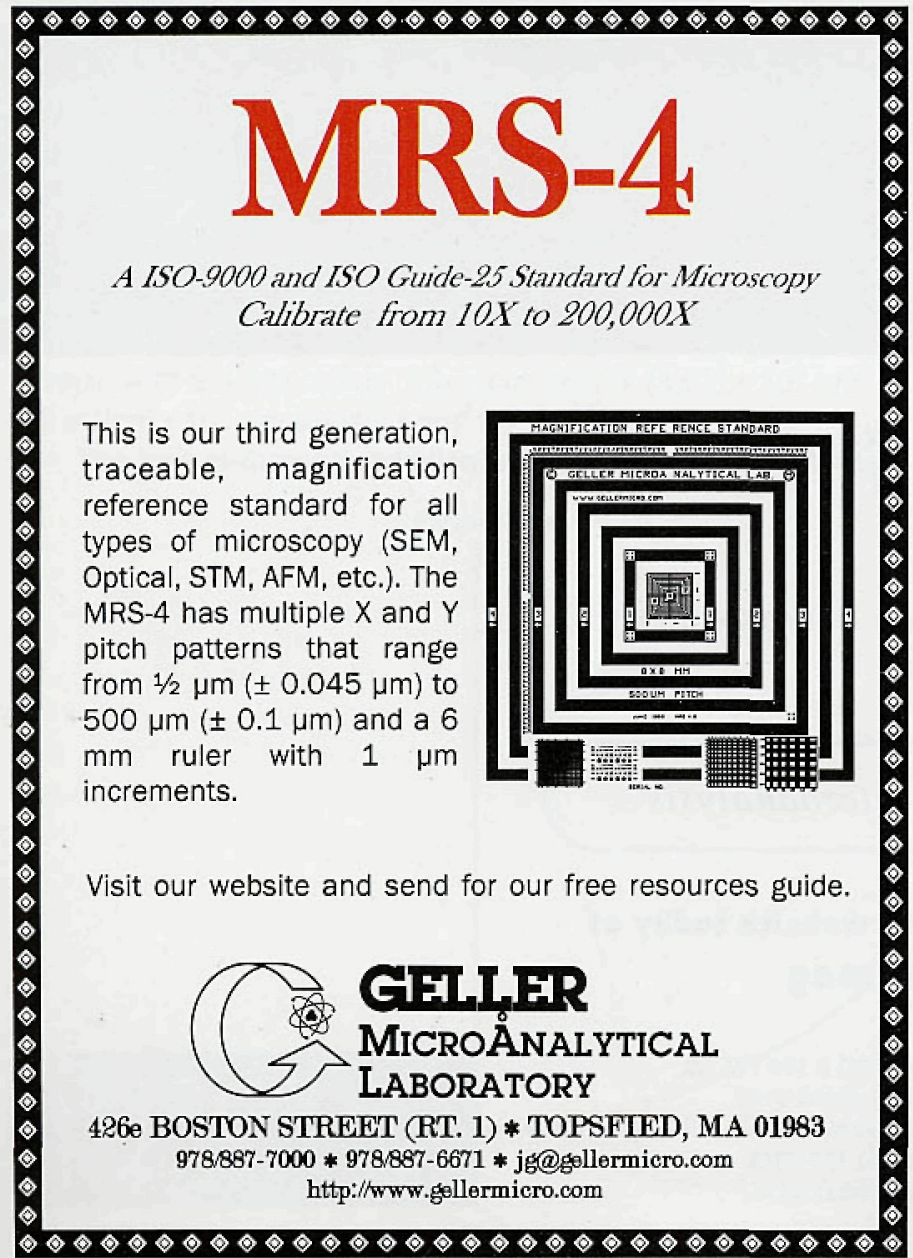

$K$ is the thermal conductivity

$\mathrm{A}_{c}$ is the appropriate contact area in $\mathrm{nm}^{2}$

$\mathrm{R}$ is in $\mathrm{nm}$

$\mathrm{J}$ is in electrons $/ \mathrm{nm}^{2}$

$T_{\mathbf{s}}$ is in Kelvins

Note that the heat capacity of the particle does not enter the equation for the steady-state temperature.

Acknowledgement

Supported by Biotechnological Resource Grant RR01219, awarded by the National Center for Research Resources, Department of Health and Human Services/Public Health Service, to support the Wadsworth Center's Biological Microscopy and Image Reconstruction Facility as a National Biotechnological Resource.

\section{References}

Glaeser, R.M. 1979. in: Introduction to Analytical Electron Microscopy . J.J. Hren, J.I. Goldstein, and D.C. Joy, eds. Plenum Press, New York and London, pg 426.

Berger, M.J. and Seltzer, S.M. 1982. Stopping Powers and Ranges of Electrons and Positrons. U.S. Department of Commerce publication NBSIR 82-2550.

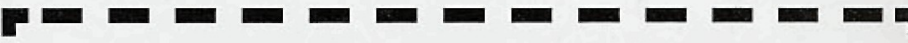 \\ University of Illinois at Chicago Research Resources Center Microscopy Specialist}

The Electron Microscopy Facility of the Research Resources Center (RRC) at the University of Illinois at Chicago has an open position for a Microscopy Specialist. The facility provides electron and laser/light microscopy services for the university research community and external organizations from two sites on the campus. The position is in the recently completed RRC-East facility which specializes in physical and materials science fields. The east side facility includes a Renishaw Raman Spectrometer, JEOL JEM-3010 and JEOL JEM-100CX TEMs, JEOL JEM-201OF and VG HB501 STEMs, and a JEOL JXA733 Microprobe.

The qualifications is minimally a bachelor's degree and preferably a master's degree or higher in physical sciences, engineering or a related field, with at least four years experience in laser and electron microscopy. This person will supervise the operation of the expanding laser microscopy area (Raman Spectroscopy and Laser Scanning Confocal Microscopy), including record keeping and maintenance and will assist the staff in the day-to-day running of the Electron Microscopes and preparation area. Interpersonal/ communications skills are important as this individual will work with users, provide technical advice and demonstrate how microscopy can advance their research.

The position is available now or until filled. Review of applications begins immediately. Send cover letter and resume listing three references, along with their addresses, to:

Alan W. Nicholls, Ph.D

Research Resources Center-East (M/C 337)

The University of Illinois at Chicago

845 West Taylor Street, Rm 100 SES

Chicago, IL 60877-7058

Via Mail, eMail: Nicholls@uic.edu, or Fax: (312)996-8091

The University of Illinois is an

Affirmative Action/Equal Opportunity Employer 


\section{O ne $B$ e a m}

M a n y

\section{on e Company.}

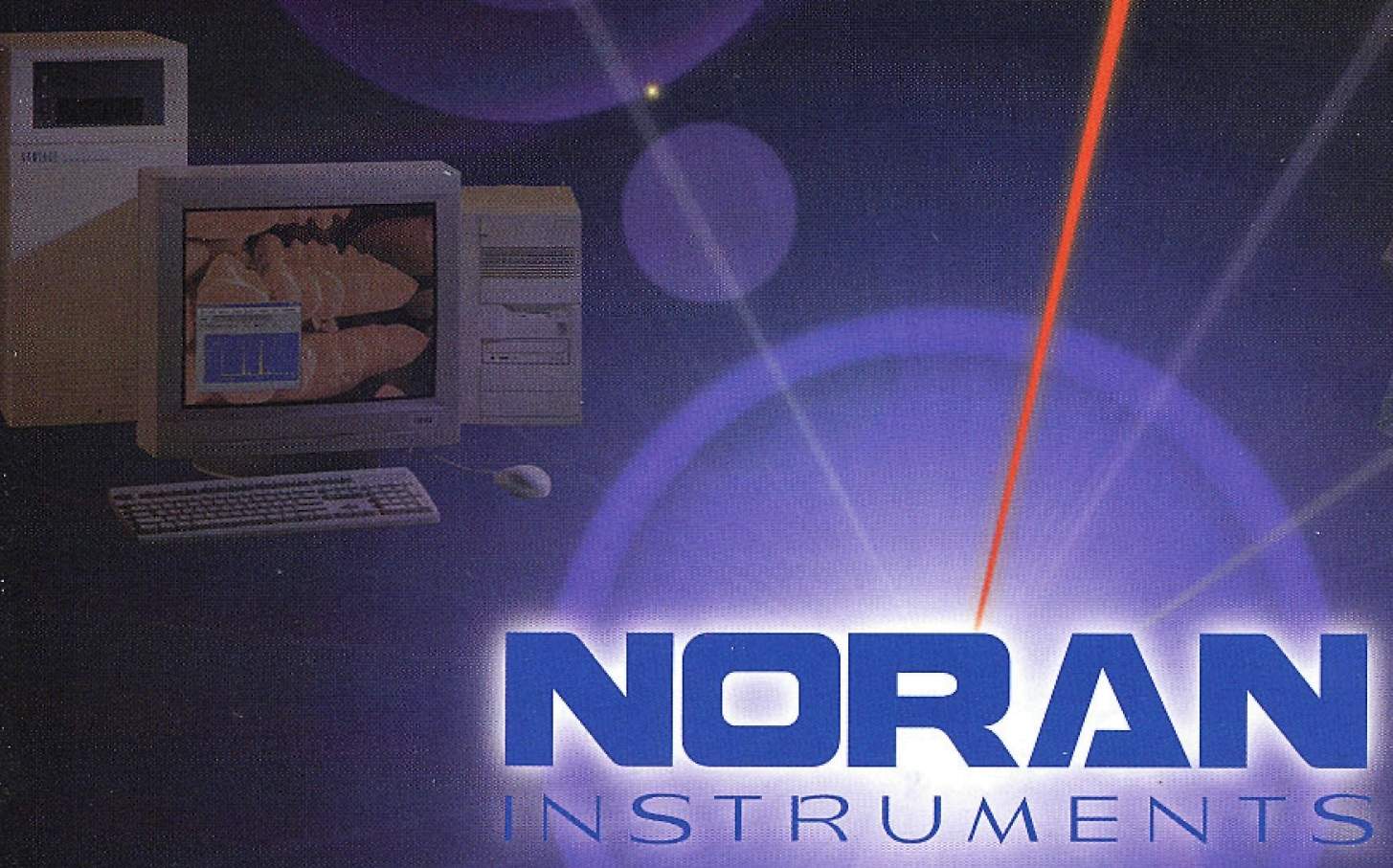

\section{Count on Us}

NORAN Instruments Ine., 2551 West Beltline Hwy., Middleton, WI 53562-2697, USA Tel.: 608831 6511, Fax: 6088367224 Internet: micro_info@noran.com, Web: http:/www.noran.com

NORAN Instruments B.V., Tel.: 3135 6998888, Fax: 31356949913

NORAN Instruments GmbH., Tel.: 497251 97910, Fax: 497251979111

WORAN Instruments Japan., Tel:: 8144246 6190, Fax: 81442466191

NORAW Instruments U.K., Tel.: 01869327711 , Fax: 01869327744 